\title{
FUNGAL SUCCESSION AND BIODEGRADTION OF RUBBER SAW DUST
}

\author{
A P Mahindadasa, M P Tissera and H S Amarasekera \\ University of Sri Jayewardenepura, \\ Nugegoda
}

Sri Lanka has a long tradition of saw milling. But the waste product (saw dust) from these mills is not adequately utilised for beneficial purposes. In a functioning sawmill about two hundred kilograms of organic matter is dispelled as saw dust everyday.

Saw dust samples were kept in the garden exposed to normal climatic conditions, a green house or on a laboratory bench for this study. Fungal species that appeared on sawdust were isolated, identified and number of fungal colonies were counted by dilution plate count technique. During this study seven prominent fungal genera were observed. Four species (Fusarium sp, Penicillium sp, Stemphylium sp. Trichodema sp) out of seven were Deuteromycetes and these were observed in all the three conditions; Two species (Lentinus $s p$ and Coprines sp) were Basidiomycetes; one (Aspergillus $s p$ ) was Ascomycetes. The fungal succession appeared in the saw dust showed that first fungal colonizers cause brown rot decay followed by white rot. Saw dust exposed to the fungal growth in laboratory conditions showed the highest weight loss $(42.86 \%)$ at the end of the study period compared with the weight loss observed in green house conditions and in laboratory conditions.

It is clear that these fungal species have the ability to degrade complex cellulose and lignin molecules to simple compounds. Due to the fungal activity, weight and strength of the saw dust were reduced, and the texture changed. This showed that there is a possibility of using saw dust as a growing medium, after its deterioration by suitable fungi.

Proceedings of the Third Annual Forestry Symposium 1997, of the Department of Forestry and Environmental Science, University of Sri Jayewardenepura, Sri Lanka 